\title{
Federico E. Turkheimer, Mattia Veronese, Joel Dunn (eds): Experimental Design and Practical Data Analysis in Positron Emission Tomography
}

\author{
CreateSpace Independent Publishing Platform, London 2014, ISBN: 978-1-49929-582-5
}

\author{
Gianluca De Matteis • Luigi Mansi
}

Published online: 13 November 2014

(C) Springer-Verlag Berlin Heidelberg 2014

This is an agile publication of 107 pages, enriched by many tables, schemata and pictures, edited by Federico E. Turkheimer, Professor of Neuroimaging at the Institute of Psychiatry, King's College, London, with Mattia Veronese and Joel Dunn, both Ph.D. research associates at the same institution. The aim of the book is to provide the reader with the ability to design, quantify and analyse a PET tracer study, supplying information on design, post-processing, general approaches to quantification and statistical analysis.

The volume is divided into 11 chapters and utilizes a graphic design combining text, images, tables and explanatory boxes. This editorial decision is effective in producing an easy read that allows accurate information on a "heavy content", but presented in a "light package", understandable also by readers who are not experts in mathematical sciences. In particular, the publication makes an interesting choice in avoiding dispersive or overly complex topics with respect to achieving the editorial aim: individuate only major issues that are analysed from many angles, from physiology to biochemistry, physics, mathematics, statistics and computer science. The basic concepts are then introduced either in an experimental or clinical scenario.

In the first two chapters, theoretical and practical bases of physiology, necessary to implement the experimental PET protocols, are treated together with elements concerning acquisition, processing and interpretation of the acquired data. To practically explain technical details and the principles on which the experiments are designed, two historical methods, i.e. $\mathrm{N}_{2} \mathrm{O}$ for the quantification of cerebral blood flow (Kety-Schmidt) and $\left[{ }^{14} \mathrm{C}\right]$ deoxyglucose to measure local glucose rate in the brain tissue (Sokoloff), are cited as examples. In the following chapter, the previously introduced principles are also applied to

G. De Matteis $\cdot$ L. Mansi $(\bowtie)$

Nuclear Medicine, Second University of Naples, Naples, Italy

e-mail: luigi.mansi@unina2.it radiological procedures. In fact, together with SPECT (the methodology of which is also applicable to PET), studies performable with functional CT and MRI are presented and discussed, after a detailed analysis of their physical-chemical bases. For an easier understanding, experimental protocols on the measurement of cerebral blood flow by $\mathrm{CT}$ with xenon or non-diffusible indicators, or, for MRI, on arterial spin labelling (ASL) phenomena and blood oxygen level-dependent (BOLD) contrast, are extensively described. The core of the book is in chapters 3-8, which deepen major aspects of PET methodology, including the physical rationale of positron-emitting radioisotopes, most frequently used radionuclides, detection instruments, mechanisms and techniques of image formation, correction and reconstruction. Particularly interesting is chapter 8 , which teaches how to correctly analyse PET by intersecting the visual examination of the images with a critical analysis of data correction for parameters such as attenuation, scatter, random events, movement and radioactive decay. The chapter is concluded with mention of the most important applications of PET in both clinical and experimental scenarios. The last three chapters are dedicated to the application of mathematical-statistical models to PET that represents the point of convergence of multiple professionals (such as physicians, technologists, physicists, chemists, mathematicians, computer scientists and so on). The exposition of the argument, in agreement with the style of the entire book, is conducted by the authors with clear, concise and appropriate language, based on text, images and graphics with immediately understandable captions, which makes the whole editorial apparatus accessible to a diverse audience.

In this sense, we suggest this book at first to college and graduate students of all faculties either clinical or "technical" who could be involved in the experimental or clinical use of PET. Great usefulness may also be found by teachers and professionals in the biomedical sector who use PET in their clinical practice or for research. 